\title{
Inhalts- und Musterverzeichnis
}

Seite

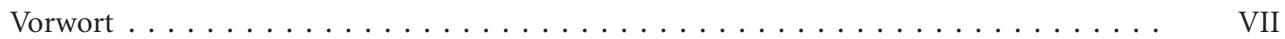

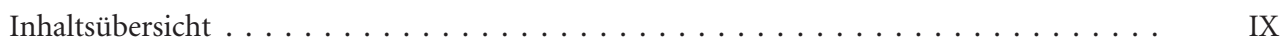

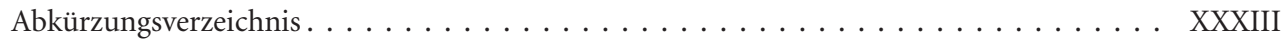

Allgemeines Literaturverzeichnis $\ldots \ldots \ldots \ldots \ldots \ldots \ldots \ldots \ldots \ldots \ldots \ldots \ldots \ldots \ldots$

Teil 1

Verträge zur Datenschutzorganisation

\section{$\$ 1$ Arbeitsvertrag mit einem Datenschutzbeauftragten}

M $1.1 \quad$ Arbeitsvertrag mit einem Datenschutzbeauftragten . . . . . . . . . . $1.7 \quad 3$

M 1.1.1 Beginn des Arbeitsverhältnisses, Arbeitsort und Aufgaben des

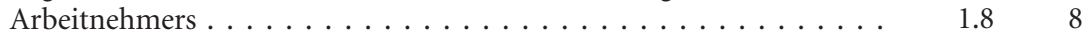

M 1.1.2 Widerruf der Bestellung zum Datenschutzbeauftragten,

Versetzungsvorbehalt . . . . . . . . . . . . . . . . $1.17 \quad 10$

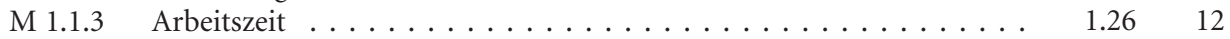

M 1.1.4 Gehalt und Aufwendungsersatz, Freiwilligkeitsvorbehalt . . . . . . . . $1.33 \quad 14$

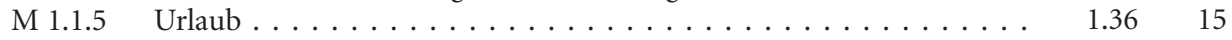

M 1.1.6 Arbeitsverhinderung und Arbeitsunfähigkeit infolge Krankheit . . . . $1.43 \quad 16$

M 1.1.7 Nebentätigkeiten, Wettbewerbsverbot . . . . . . . . . . . . . . . $1.45 \quad 17$

M 1.1.8 Arbeitsergebnisse, Erfindungen, Urheberrecht . . . . . . . . . . . . . $1.48 \quad 18$

M 1.1.9 Laufzeit und Beendigung des Arbeitsverhältnisses . . . . . . . . . . . . $1.50 \quad 18$

M 1.1.10 Verschwiegenheitspflicht, Herausgabe von Gegenständen . . . . . . . . 1.6121

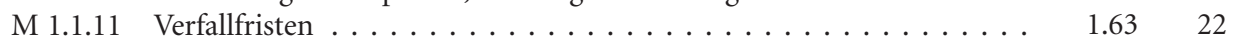

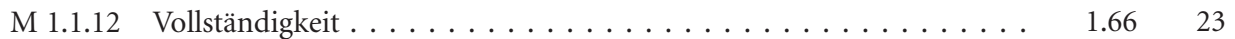

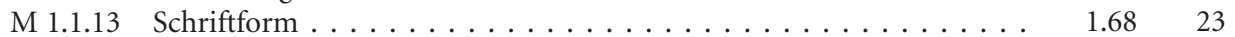

M 1.1.14 Salvatorische Klausel . . . . . . . . . . . . . . . . . . . . . . 1.7124

M 1.2 Ergänzungsvertrag zum Arbeitsvertrag (Bestellung zum Datenschutz-

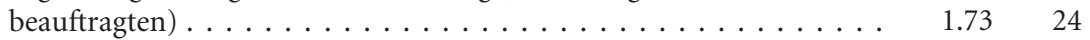

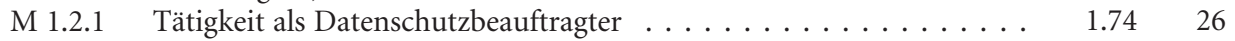

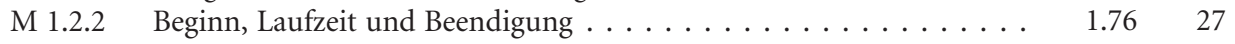

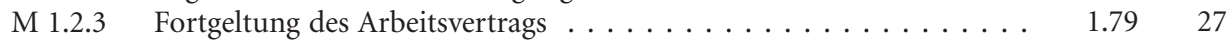

M 1.2.4 Vollständigkeit, Schriftform, Salvatorische Klausel . . . . . . . . . 1.8127

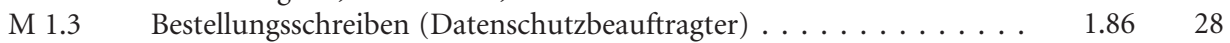

\section{$\$ 2$ Vertrag über die Beauftragung eines externen Datenschutzbeauftragten}

M $2.1 \quad$ Vertrag über die Beauftragung eines externen Datenschutz-

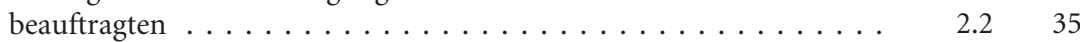

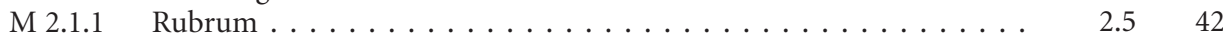

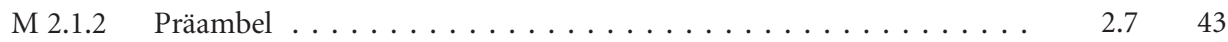

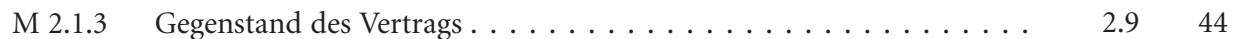

M 2.1.4 Organisatorisches zur Einbindung des Auftragnehmers . . . . . . . $2.12 \quad 45$

M 2.1.5 Zeit, Ort und Personal zur Leistungserbringung . . . . . . . . . . . . $2.19 \quad 247$

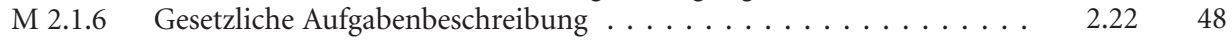

M 2.1.7 Konkretisierung der gesetzlichen Aufgaben . . . . . . . . . . . $2.24 \quad 48$ 


\begin{tabular}{|c|c|c|}
\hline & & Rz. \\
\hline M 2.1.8 & Umfang der Beratungs- und Prüfpflichten & 2.36 \\
\hline M 2.1.9 & Fachwissen und Fehlen von Interessenkollisionen & 2.38 \\
\hline M 2.1.10 & Verschwiegenheitspflicht . . . . . . . . . . . & 2.42 \\
\hline M 2.1.11 & Vergütung & 2.50 \\
\hline M 2.1.12 & Haftung & 2.56 \\
\hline M 2.1.13 & Vertragslaufzeit und Abberufung & 2.60 \\
\hline M 2.1.14 & Schlussbestimmungen .... & 2.66 \\
\hline
\end{tabular}

\section{$\$ 3$ Vertrag über die Beauftragung eines Vertreters in der EU}

M 3.1 Vertrag über die Beauftragung eines Vertreters in der EU . . . . . . . .

M 3.2 Agreement on the designation of a representative in the EU . . . . .

M 3.1.1 Bestellung, Funktion und Vertretungsbefugnisse . . . . . . . . . .

M 3.1.2 Einrichtung einer Anlaufstelle . . . . . . . . . . . . . . .

M 3.1.3 Verzeichnis der Verarbeitungstätigkeiten . . . . . . . . . . . .

\section{$\$ 4$ Geheimhaltungsvereinbarung}

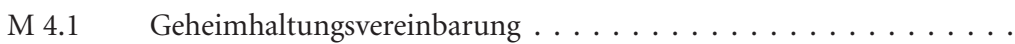

Non Disclosure Agreement . . . . . . . . . . . . . . .

\section{\$5 Vereinbarung zwischen gemeinsam Verantwortlichen}

M 5.1 Vereinbarung zwischen gemeinsam Verantwortlichen . . . . . . . .

Joint Control Agreement . . . . . . . . . . . . . . . .

M 5.1.2 Gegenstand, Zweck, Mittel und Umfang der Datenverarbeitung . . .

M 5.1.3 Phasen der Datenverarbeitung/Zuständigkeiten und Verantwortung . Information der betroffenen Personen . . . . . . . . . . . 


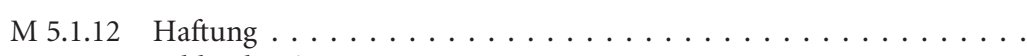

Rz. Seite

M 5.1 .13 Schlussbestimmungen ...................

$5.116 \quad 148$

$5.126 \quad 151$

\section{$\$ 6$ Vertrag zur Durchführung eines Datenschutzaudits}

M 6.1 Vertrag zur Durchführung eines Datenschutzaudits . . . . . . . .

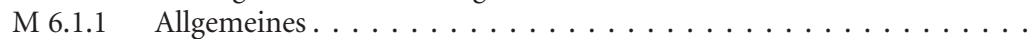

M 6.1.2 Gegenstand des Audits . . . . . . . . . . . . . . . .

6.19164

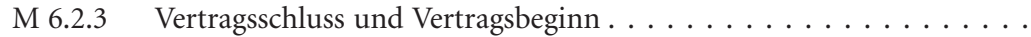

6.27166

M 6.1.4 Leistungen des Auftragnehmers . . . . . . . . . . . . . . .

6.31166

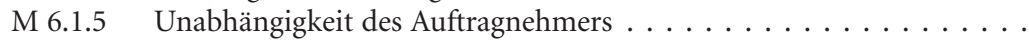

6.42168

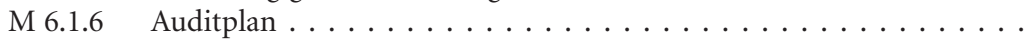

6.46169

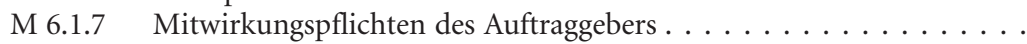

$6.58 \quad 171$

M 6.1.8

Abschlussbesprechung und Prüfbericht . . . . . . . . . . . . . . . . . . . . . . . . . . . . . . . . . . . . .

6.69

6.78

M 6.1.9

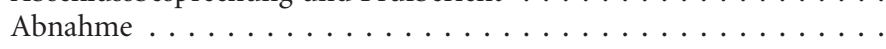

175

M 6.1.10

Zertifikat und Nutzungsrechte . . . . . . . . . . . .

6.86

M 6.1.11

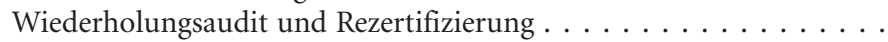

6.101

M 6.1.12

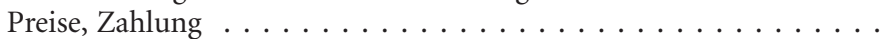

6.105

M 6.1.13

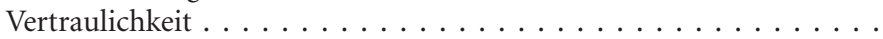

6.110

M 6.1.14

Datenschutz

6.122

M 6.1.15

Gewährleistung und Verzug. . . . . . . . . . . . . . . . . .

6.127

6.136

M 6.1.17

Haftungsbeschränkung

6.143

M 6.1.18

Vertragsdauer/Kündigung . . . . . . . . . . . . . . .

6.147

\section{Teil 2}

\section{Datenschutzverträge}

\section{$\$ 7$ Kurzer Auftragsverarbeitungsvertrag (Anlage)}

M $7.1 \quad$ Kurzer Auftragsverarbeitungsvertrag (Anlage) . . . . . . . . . . . . . .

Alternativformulierung für den Anwendungsbereich des Auftrags-

M 7.1.3 Auftragsgegenstand und Weisungsbefugnis $\ldots \ldots \ldots \ldots \ldots \ldots \ldots \ldots$

Anforderungen an Personal . . . . . . . . . . . . . . . . .

Sicherheit der Verarbeitung . . . . . . . . . . . . . . . . . . . . . .

\section{\$8 Allgemeiner Auftragsverarbeitungsvertrag - auftraggeberfreundlich}

M 8.1 Auftragsverarbeitungsvertrag (auftraggeberfreundlich) . . . . . . .

M 8.2 Data Processing Agreement (customer-friendly) . . . . . . . . . .

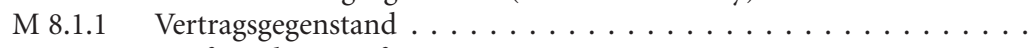


M 8.1.4

M 8.1.5

M 8.1.6

M 8.1.7

M 8.1.8

M 8.1.9

M 8.1.10

M 8.1.11

M 8.1.12

M 8.1.13

M 8.1.14

M 8.1.15

Rechtsstellung des Auftraggebers . . . . . . . . . . . . . . . . .

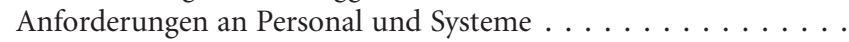

8.47258

Sicherheit der Verarbeitung . . . . . . . . . . . . . .

$8.51 \quad 259$

Inanspruchnahme weiterer Auftragsverarbeiter $\ldots \ldots \ldots \ldots$

8.56261

Rechte der betroffenen Personen . . . . . . . . . . . . .

8.71265

8.89270

bei Verletzungen des Schutzes von Auftraggeber-Daten . . . . . . .

$8.98 \quad 272$

Sonstige Unterstützungspflichten des Auftragnehmers . . . . . . . . . . . . . . . . . . . . . . . . . . . .

8.111275

8.121278

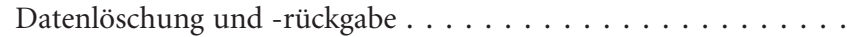

8.131280

Nachweise und Überprüfungen $\ldots \ldots \ldots \ldots \ldots$

8.153286

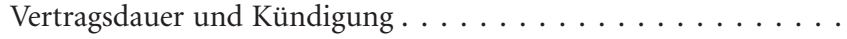

$8.160 \quad 288$

Haftung und Vertragsstrafe $\ldots \ldots \ldots \ldots \ldots$

$8.176 \quad 291$

\$9 Allgemeiner Auftragsverarbeitungsvertrag - auftragnehmerfreundlich

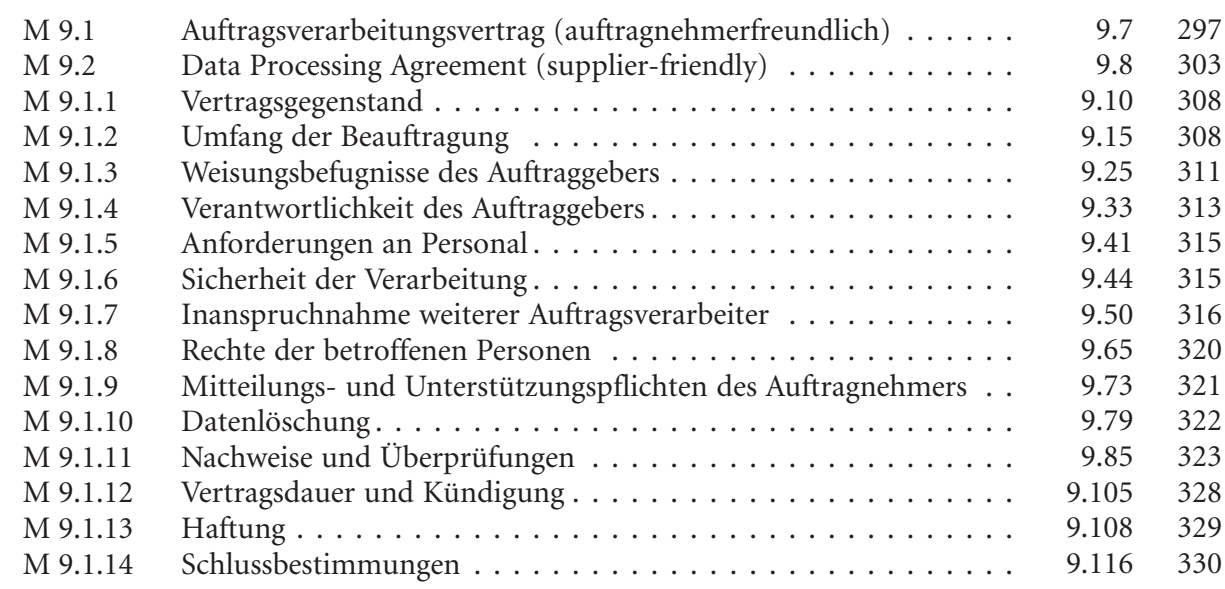

\section{$\$ 10$ Datenschutzklausel Outsourcingvertrag}

M $10.1 \quad$ Datenschutzklausel Outsourcingvertrag . . . . . . . . . . .

M 10.1.1

Datenschutzklausel für einen Outsourcingvertrag . . . . . . . . . . . . .

M 10.1.2

Change Management-Regelung Outsourcingvertrag . . . . . . . . .

10.15343

M 10.1.3

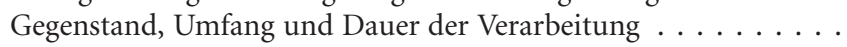

$10.16 \quad 344$

M 10.1.4

Weisungen des Auftraggebers . . . . . . . . . . . . . . . . . . . .

$10.20 \quad 345$

M 10.1.5

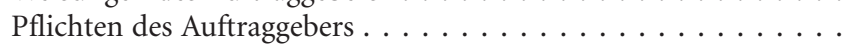

10.28

M 10.1.6

Allgemeine Organisationspflichten, technische und

organisatorische Maßnahmen . . . . . . . . . . . . . . . . . 10.32

M 10.1.7

Einschaltung weiterer Auftragsverarbeiter . . . . . . . . . . . . . . .

10.38

349

M 10.1.8

Subunternehmer-Klausel für Vertragsanlage Datenschutz . . . . . .

10.42

350

M 10.1.9

Rechte der betroffenen Person . . . . . . . . . . . . . . . . . . . . .

$10.43 \quad 350$

M 10.1.10

Meldung von Datenschutzverstößen $\ldots \ldots \ldots \ldots$

$10.45 \quad 351$

M 10.1.11

Datenschutz-Folgenabschätzungen und vorherige Konsultationen

10.48

352

M 10.1.12

Herausgabe- und Löschungspflichten bei Beendigung des

M 10.1.13

Auftrags . . . . . . . . . . . . . . . . .

10.50

352

$10.53 \quad 353$ 
M 10.1.14 Alternativ-Regelung für Prüfungen des Auftraggebers

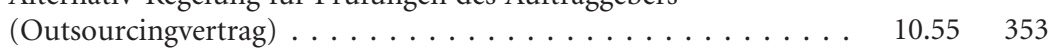

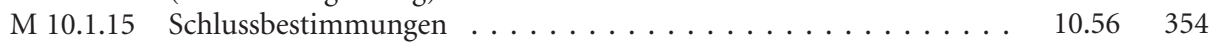

\section{$\$ 11$ Vertrag zur Datenträgervernichtung/-löschung}

M $11.1 \quad$ Vertrag zur Datenträgervernichtung/-löschung . . . . . . . . . . . . $11.5 \quad 357$

M 11.1.1 Präambel. . . . . . . . . . . . . . . . . . . . . 11.11363

M 11.1.2 Gegenstand des Auftrags und allgemeine Pflichten. . . . . . . . . . . 11.16364

M 11.1.3 Vernichtung und/oder Löschung von Datenträgern . . . . . . . . . . $11.23 \quad 366$

M 11.1.4 Dauer des Auftrags . . . . . . . . . . . . . . . . . . . . . . 11.3937

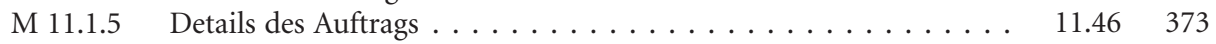

M 11.1.6 Weisungsrechte . . . . . . . . . . . . . . . . . . . . $11.50 \quad 374$

M 11.1.7 Drittstaatentransfer . . . . . . . . . . . . . . . . . . . 11.55375

M 11.1.8 Vertraulichkeit . . . . . . . . . . . . . . . . 11.59376

M 11.1.9 Technische und organisatorische Maßnahmen . . . . . . . . . . 11.64378

M 11.1.10 Unterauftragsverhältnisse . . . . . . . . . . . . . . . . $11.71 \quad 379$

M 11.1.11 Informations- und Unterstützungspflichten . . . . . . . . . . . . 11.79381

M 11.1.12 Rückgabe von Datenträgern . . . . . . . . . . . . . . . . . . . $11.86 \quad 383$

M 11.1.13 Prüfungs- und Kontrollhandlungen . . . . . . . . . . . . . . . 11.89384

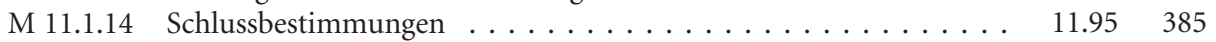

\section{$\$ 12$ Datentreuhändervertrag}

M 12.1 Datentreuhändervertrag - Vertragspartei als Datentreuhänder/

M $12.2 \quad$ Verhältnis zwischen den Vertragsparteien . . . . . . . . . . . . . . . .

M 12.3 Datentreuhändervertrag - Generelles Offenlegungsverbot . . . . . . .

M 12.1.1 Auskunfts- und Offenlegungspflichten . . . . . . . . . . . .

M 12.1.2

Offenlegung von Daten/Voraussetzungen $\ldots \ldots \ldots \ldots \ldots$

$12.52 \quad 402$

M 12.2.1

Präambel. . . . . . . . . . . . . . . . . .

$12.64 \quad 404$

M 12.2.2

Umfang der Beauftragung . . . . . . . . . . . . .

$12.67 \quad 405$

M 12.2.3

Verwahrung und Offenlegung von Daten $\ldots \ldots \ldots \ldots \ldots$

$12.71 \quad 405$

M 12.2.4

Auskunftsrecht des Auftraggebers $\ldots \ldots \ldots \ldots \ldots \ldots \ldots \ldots \ldots \ldots$
Umfang der Beauftragung $\ldots \ldots \ldots \ldots \ldots \ldots \ldots \ldots \ldots$

$12.75 \quad 407$

M 12.3.1

12.82

M 12.3.2

Pflichten des Datentreuhänders.

12.85

408

408

\section{Teil 3}

\section{Datennutzungsverträge}

\section{$\$ 13$ Auskunfteivertrag}

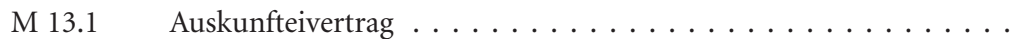

M 13.1.1 Rubrum eines Auskunfteivertrags . . . . . . . . . . . .

M 13.1.2 Präambel eines Auskunfteivertrags . . . . . . . . . . . .

$13.6 \quad 421$

M 13.1.3 Vertragsgegenstand eines Auskunfteivertrags . . . . . . . . . .

$13.8 \quad 421$

M 13.1.4

Datenbestand der Auskunftei . . . . . . . . . . . . . . . . .

$13.13 \quad 422$

M 13.1.5

Datenschutz . . . . . . . . . . . . . . . . .

$13.15 \quad 424$

M 13.1.6

Vorliegen und Nachweis des berechtigten Interesses . . . . . . . . .

$13.24 \quad 427$

M 13.1.7

Identität/Nutzungsverbot $\ldots \ldots \ldots \ldots \ldots \ldots$

13.28

M 13.1.8

Leistungsübergabepunkt

13.31

429

430 


\begin{tabular}{|c|c|c|}
\hline & & Rz. \\
\hline M 13.1.9 & Dauer der Datenspeicherung & 13.33 \\
\hline M 13.1.10 & Processingunternehmen/Erfüllungsgehilfen . . . . . . . . . & 13.35 \\
\hline M 13.1.11 & Pflichten des Datenempfängers $\ldots \ldots \ldots \ldots \ldots \ldots$ & 13.37 \\
\hline M 13.1.12 & Profilbildung (Scoring) & 13.43 \\
\hline M 13.1.13 & Intervall der Leistungserbringung . & 13.49 \\
\hline M 13.1.14 & Vergütung & 13.51 \\
\hline M 13.1.15 & Laufzeit und Kündigung/Inkrafttreten . & 13.53 \\
\hline M 13.1.16 & Kommunikationsverfahren . . . . . . . . . . . & 13.55 \\
\hline M 13.1.17 & Haftung $\ldots \ldots \ldots \ldots \ldots \ldots$ & 13.57 \\
\hline M 13.1.18 & Informationsweitergabe und Geheimhaltung $\ldots \ldots \ldots \ldots$ & 13.66 \\
\hline M 13.1.19 & Ansprechpartner $\ldots \ldots \ldots \ldots \ldots \ldots$ & 13.68 \\
\hline M 13.1.20 & 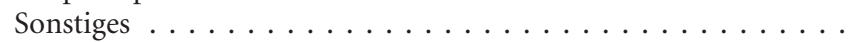 & 13.70 \\
\hline
\end{tabular}

\section{$\$ 14$ Adressenkauf- und -überlassungsvertrag}

M $14.1 \quad$ Adressenlieferungsrahmenvertrag . . . . . . . . . . . . . .

M 14.1.1 Gegenstand des Vertrags. . . . . . . . . . . . . . . .

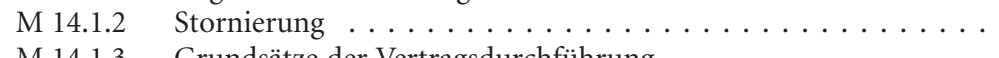

Grundsätze der Vertragsdurchführung . . . . . . . . . . . . .

$14.18 \quad 457$

M 14.1.4

M 14.1.5

Unterauftragnehmer . . . . . . . . . . . . . . . .

$14.21 \quad 458$

M 14.1.6

Datenqualität in tatsächlicher und rechtlicher Hinsicht . . . . . . .

$14.24 \quad 459$

M 14.1.7

Datenabgleich und Abrechnung ,netto erreicht"; Vergütung . . . .

$14.42 \quad 467$

M 14.1.8

Gewährleistung . . . . . . . . . . . . . . .

$14.48 \quad 469$

M 14.1.9

Haftung des Lieferanten und Freistellung . . . . . . . . . . . . . . .

$14.50 \quad 470$

$14.55 \quad 472$

\section{$\S 15$ Datenlieferungsvertrag}

M 15.1

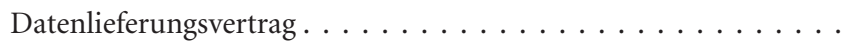

15.6

15.14

M 15.1.1

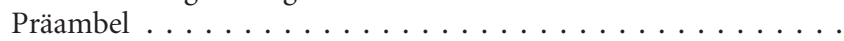

15.18

475

M 15.1.2

Vertragsgegenstand . . . . . . . . . . . . . . . .

15.18483

M 15.1.3

Bereitstellen der Lieferanten-Datensätze . . . . . . . . . . . . . . . . . .

15.27

M 15.1.4

Nutzungsrechte an den Lieferanten-Datensätzen . . . . . . . . . . . . . . . . . . . . . . . . . . . . . . . . . . . . . . . .

15.31

15.49

M 15.1.5

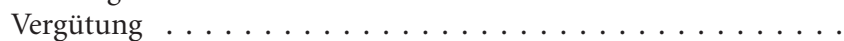

15.52

M 15.1.6

M 15.1.7

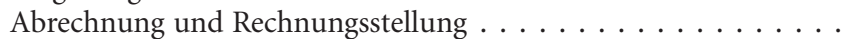

15.59
15.63

M 15.1.8

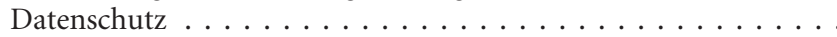

M 15.1.9

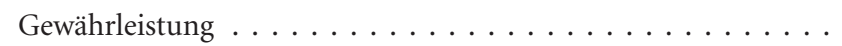

15.63
15.75

M 15.1.10

Haftung und Freistellung . . . . . . . . . . . . .

15.75
15.81

M 15.1.11

Vertragsstrafe und Informationspflicht

15.81

Vertragsdauer, -beendigung und Datenlöschung. . . . . . . . . . 500

\section{$\$ 16$ Marktforschungsvertrag/Social Media Monitoring-Vertrag}

M 16.1 Marktforschungsvertrag/Social Media Monitoring-Vertrag . . . . .

M 16.1.1

M 16.1.2

M 16.1.3

M 16.1.4

M 16.1.5

M 16.1.6

M 16.1.7

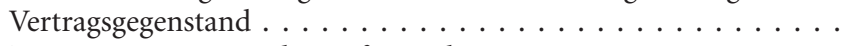
Zugang zum System des Auftragnehmers . . . . . . . . . . . . . . . .

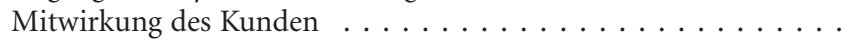

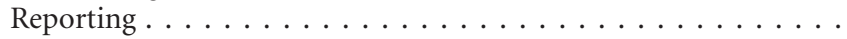

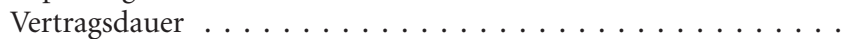

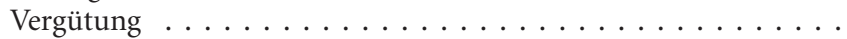
Keine Rechte des Kunden an Daten .
$16.11 \quad 510$

$16.24 \quad 513$

$16.26 \quad 513$

$16.28 \quad 514$

$16.31 \quad 514$

$16.33 \quad 515$

$16.35 \quad 515$ 


\begin{tabular}{|c|c|c|}
\hline M 16.1.8 & en Ergebnissen. . . . . . . . . & 16.38 \\
\hline & keit & 16.41 \\
\hline & & 16.43 \\
\hline & · & 16.45 \\
\hline & & 16.47 \\
\hline & Ho & 16.51 \\
\hline 16.1 .14 & Schlussbestimmungen & 16.53 \\
\hline
\end{tabular}

\section{$\$ 17$ Vertrag zur Durchführung von Webanalysen („Webtracking“)}

M 17.1 Vertrag zur Durchführung von Webanalysen („Webtracking“) . . $\quad 17.24 \quad 528$

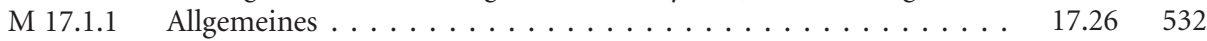

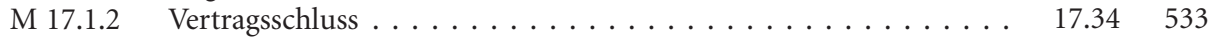

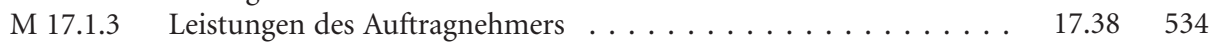

M 17.1.4 Pflichten des Auftraggebers . . . . . . . . . . . . . . . . 17.49536

M 17.1.5 Datenschutz . . . . . . . . . . . . . . . . . . . . . . . . . 17.61538

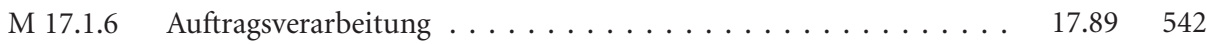

M 17.1.7 Nutzungsrechte. . . . . . . . . . . . . . . . . . . . . . . $17.94 \quad 542$

M 17.1.8 Datensicherung. . . . . . . . . . . . . . . . . . . . . . . . . . . . . 17.9954

M 17.1.9 Preise und Zahlung . . . . . . . . . . . . . . . . . . . . . . . . . . . . 17.10454

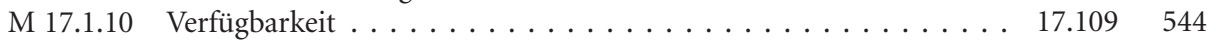

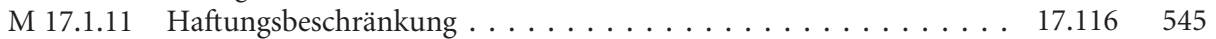

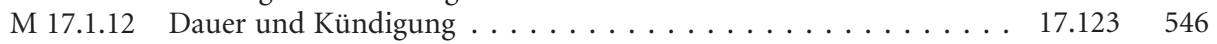

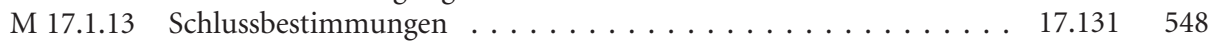

\section{$\$ 18$ Datenbanklizenz}

M 18.1 Datenbanklizenz . . . . . . . . . . . . . . . . .

M 18.1.1

Geltungsbereich . . . . . . . . . . . . . .

18.8

M 18.1.2

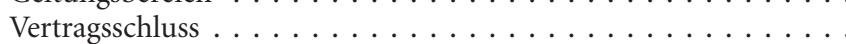

18.17

18.23

M 18.1.3

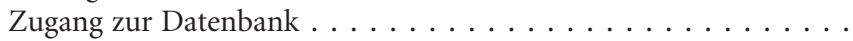

560

M 18.1.5

Informationen für Verbraucher .

$18.30 \quad 562$

M 18.1.6

Vorausgesetzte Hard- und Software

18.35

$18.39 \quad 565$

M 18.1.7

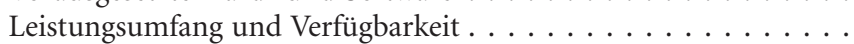

$18.43 \quad 566$

M 18.1.8

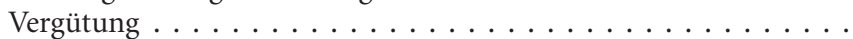

$18.49 \quad 568$

M 18.1.9

Nutzungsrechte. . . . . . . . . . . . . . .

$18.55 \quad 570$

M 18.1.10

Datenschutz . . . . . . . . . . . . . . .

$18.57 \quad 571$

M 18.1.11

Gewährleistung und Haftung . . . . . . . . . . . .

$18.61 \quad 572$

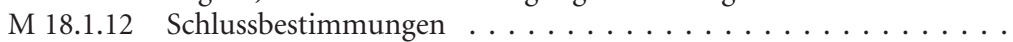

18.66

M 18.1.13

Stand der Nutzungsbedingungen .

18.71

573 573

\section{Teil 4}

\section{Unternehmensrichtlinien und Betriebsvereinbarungen}

\section{$\$ 19$ Unternehmensrichtlinie Datenschutz}

M 19.1 Unternehmensrichtlinie Datenschutz . . . . . . . . . . .

M 19.1.1 Einleitung . . . . . . . . . . . . . . . . . 
M 19.1.4

M 19.1.5

M 19.1.6

M 19.1.7

M 19.1.8

M 19.1.9

M 19.1.10

M 19.1.11

M 19.1.12

M 19.1.13

M 19.1.14

M 19.1.15

M 19.1.16

M 19.1.17

M 19.1.18

M 19.1.19

M 19.1.20

M 19.1.21

M 19.1.22

M 19.1.23

M 19.1.24

M 19.1.25

M 19.1.26

M 19.1.27

M 19.1.28

M 19.1.29

M 19.1.30

M 19.1.31

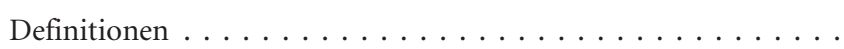

Zulässigkeit der Datenverarbeitung . . . . . . . . . . .

Gesetzliche Ermächtigungsgrundlagen . . . . . . . . . . . . .

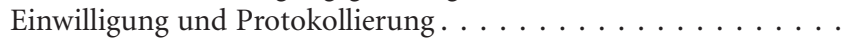

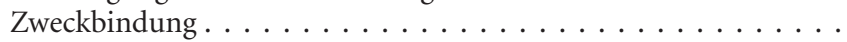

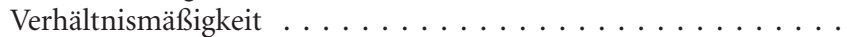

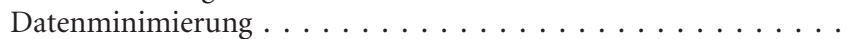

Direkterhebung und Information des Betroffenen . . . . . . . .

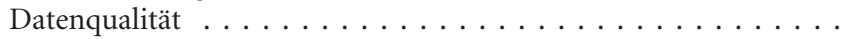

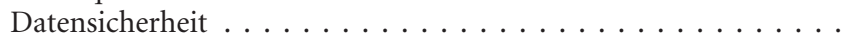

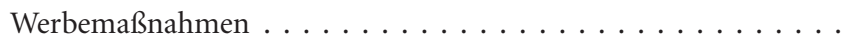

Erstellung von Nutzerprofilen . . . . . . . . . . . . . . . . .

Verarbeitung besonderer Arten von Daten . . . . . . . . . .

Auftragsverarbeitung . . . . . . . . . . . . .

Automatisierte Einzelentscheidungen $\ldots \ldots \ldots \ldots \ldots$

Übermittlung von Daten . . . . . . . . . . . . . . . .

Anforderungen an Mitarbeiter . . . . . . . . . . . . .

Dokumentationspflichten . . . . . . . . . . . . .

Einführung neuer Systeme zur Datenverarbeitung . . . . . . . .

Recht auf Auskunft und Datenübertragbarkeit . . . . . . . . . . .

Löschung und Einschränkung der Verarbeitung . . . . . . . . . . .

Recht auf Berichtigung . . . . . . . . . . . . .

Recht auf Widerruf, Widerspruch und Beschwerde . . . . . . . .

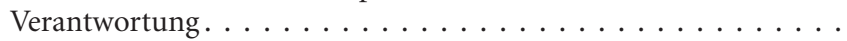

Datenschutzbeauftragter als Ansprechpartner . . . . . . . . .

Meldung von Verstößen und Zusammenarbeit mit Aufsichts-

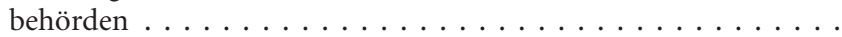

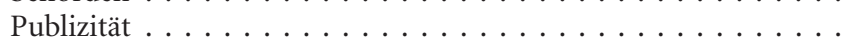

Änderungen dieser Unternehmensrichtlinie
Rz. Seite

$19.35 \quad 595$

$19.44 \quad 597$

$19.48 \quad 598$

$19.56 \quad 600$

$19.68 \quad 602$

$19.74 \quad 603$

$19.83 \quad 605$

$19.92 \quad 607$

$19.97 \quad 608$

$19.102 \quad 609$

$19.106 \quad 610$

$19.112 \quad 611$

$19.118 \quad 613$

$19.128 \quad 615$

$19.137 \quad 617$

$19.144 \quad 618$

$19.149 \quad 619$

$19.154 \quad 620$

$19.163 \quad 622$

$19.167 \quad 623$

$19.180 \quad 626$

$19.187 \quad 628$

$19.191 \quad 628$

$19.200 \quad 630$

$19.206 \quad 632$

$19.215 \quad 634$

$19.226 \quad 637$

$19.232 \quad 638$

\section{$\$ 20$ Betriebsvereinbarung zur Videoüberwachung}

M $20.1 \quad$ Betriebsvereinbarung zur Videoüberwachung . . . . . . . .

M 20.1.1 Bezeichnung der Vertragsparteien und Präambel . . . . . . . . . .

M 20.1.2 Geltungsbereich . . . . . . . . . . . . . . . .

$20.38 \quad 653$

M 20.1.3

Zweck der Videoüberwachung . . . . . . . . . . . . . . . .

$20.43 \quad 654$

M 20.1.4

M 20.1.5

Erfasste Bereiche, Einsatzform und Kenntlichmachung . . . . . . .

20.50

655

M 20.1.6

Dokumentation . . . . . . . . . . . . . . .

20.73

661

M 20.1.7

Übermittlung der Bilder und Schnittstellen . . . . . . . . . . . . .

20.78

20.81

M 20.1.8

Aufzeichnung und Speicherung . . . . . . . . . . . .

Zugriff auf Aufzeichnungen und Auswertung . . . . . . . . . 20.92

M 20.1.9

Rechte der Arbeitnehmer . . . . . . . . . . . . . . . .

20.97

M 20.1.10 Zutritt zu Räumen mit Komponenten des Videoüberwachungs-

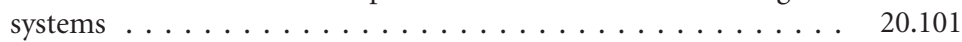

662

663

666

668

M 20.1.11 Änderungen am Videoüberwachungssystem . . . . . . . . . . . . . . . . 20.107

669

M 20.1.12

Schlussbestimmungen .

20.110

670

671

\section{$\$ 21$ Betriebsvereinbarung zur Mitarbeiterortung}

M $21.1 \quad$ Betriebsvereinbarung zur Mitarbeiterortung . . . . . . . . 
M 21.1.2 Anwendungsbereich . . . . . . . . . . . . . . . . . . . . . . 21.31684

M 21.1.3 Einführung einer Ortungseinrichtung . . . . . . . . . . . . . . . . . . . . . . . 21.38685

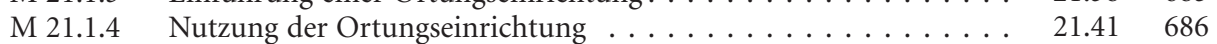

M 21.1.5 Speicherung, Löschung und Sperrung von Daten _ . . . . . . . . . . 21.51689

M 21.1.6 Änderungen und Erweiterungen der Ortungseinrichtung . . . . . $21.55 \quad 689$

M 21.1.7 Information der Arbeitnehmer; Ansprechpartner . . . . . . . . . 21.57690

M 21.1.8 Meinungsverschiedenheiten . . . . . . . . . . . . . . . . . . . . . 21.61690

M 21.1.9 Beginn, Laufzeit und Beendigung . . . . . . . . . . . . . . . . 21.63691

\section{\$22 Whistleblowing-Richtlinie}

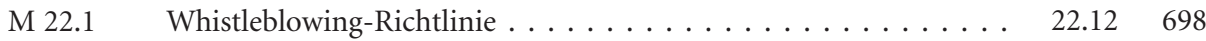

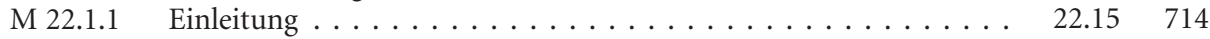

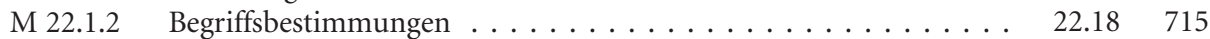

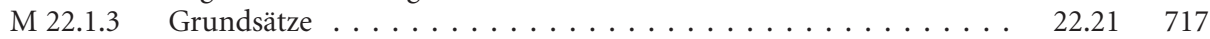

M 22.1.4 Meldeverfahren . . . . . . . . . . . . . . . . . . . . . . 22.39723

M 22.1.5 Verfahrensablauf nach Meldung . . . . . . . . . . . . . . . . 22.62731

M 22.1.6 Schutz des Hinweisgebers und der bei der Aufklärung

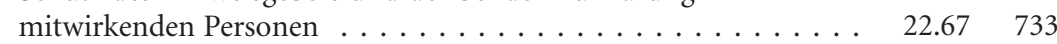

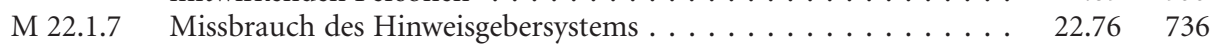

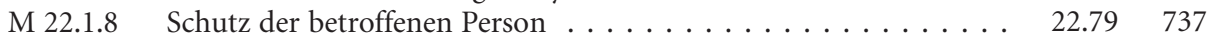

M 22.1.9 Rechte der Betroffenen . . . . . . . . . . . . . . . . . . . . . . . . . . . . . . . . . . . . . . 740

M 22.1.10 Widerspruchsrechte . . . . . . . . . . . . . . . . . . . 22.98742

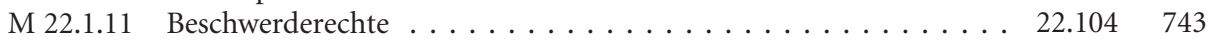

M 22.1.12 Datenschutz . . . . . . . . . . . . . . . . . . . . . . . . . 22.110745

M 22.1.13 Umsetzung/Verantwortlichkeit . . . . . . . . . . . . . . . . . . . . 22.119748

M 22.1.14 Informationen, Schulungen, Ansprechpartner . . . . . . . . . . . 22.123749

\section{$\$ 23$ Betriebsvereinbarung zur Internet- und E-Mail-Nutzung}

M 23.1 Betriebsvereinbarung zur Internet- und E-Mail-Nutzung . . . . 23.4754

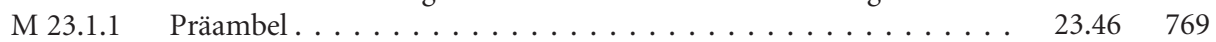

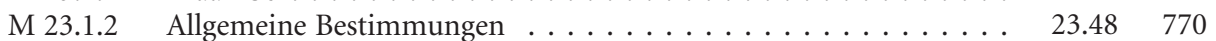

M 23.1.3 Grundregeln zur Nutzung von E-Mail- und Internet-Accounts . . . 23.54771

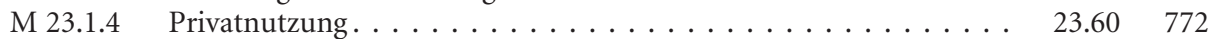

M 23.1.5 Spezielle Regeln für E-Mail-Accounts . . . . . . . . . . . . . . . . . . . . . . . . . . . . . . . 7576

M 23.1.6 Spezielle Regeln für Internet-Accounts . . . . . . . . . . . . . . . . 23.95780

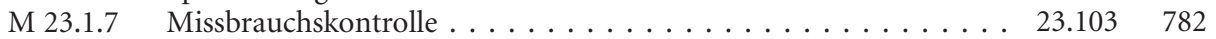

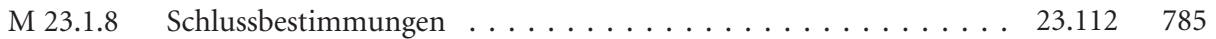

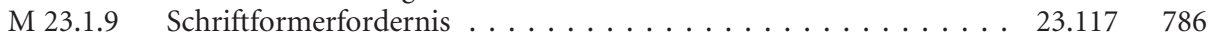

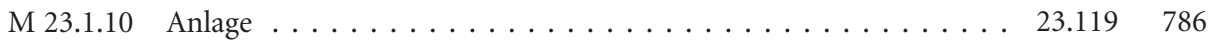

\section{$\$ 24$ Betriebsvereinbarung zu Bring Your Own Device (BYOD)}

M $24.1 \quad$ Betriebsvereinbarung zu Bring Your Own Device . . . . . . . . . 24.5798

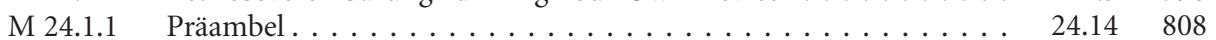

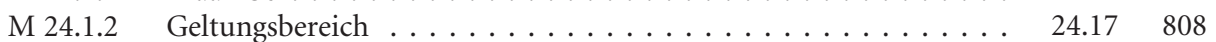

M 24.1.3 Nutzung privater mobiler Geräte . . . . . . . . . . . . . . . . . . . . 24.20809

M 24.1.4 Sicherheitsmaßnahmen . . . . . . . . . . . . . . . . . . . . . $24.30 \quad 812$

M 24.1.5 Datenspeicherung und Zugang zu Daten . . . . . . . . . . . . . . . . 24.39814

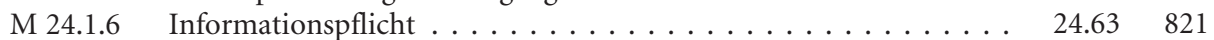




\begin{tabular}{|c|c|c|}
\hline & & \\
\hline M 24.1.7 & Nutzung von Kommunikationsdiensten $\ldots \ldots \ldots \ldots \ldots \ldots \ldots$ & 24.69 \\
\hline & astallation von Apps $\ldots \ldots \ldots \ldots \ldots \ldots \ldots$ & 24.72 \\
\hline 24.1 .9 & 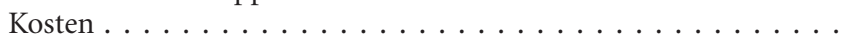 & 24.78 \\
\hline 24.1.10 & Haftung . & 24.84 \\
\hline 24.1.11 & Arbe & 24.91 \\
\hline 24.1.12 & tlichen Nutzung & 24.95 \\
\hline 24.1.13 & Her & 24.98 \\
\hline 1.14 & Erlaubnisnor & 24.103 \\
\hline 24.1.15 & 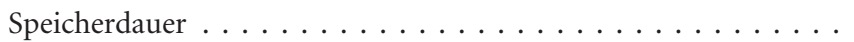 & 24.109 \\
\hline 6 & Bet & 24.117 \\
\hline 7 & Sal & 24.121 \\
\hline 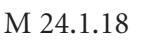 & Geltungsdauer & 24.123 \\
\hline 24.1.19 & Einwilligungserklärung & 24.125 \\
\hline
\end{tabular}

\section{$\$ 25$ Social Media-Richtlinie}

M $25.1 \quad$ Social Media-Richtlinie $\ldots \ldots \ldots \ldots \ldots \ldots \ldots$

M 25.1.1 Eigenverantwortung des Arbeitnehmers . . . . . . . . . . . . .

M 25.1.2 Keine Erklärungen für den Arbeitgeber . . . . . . . . . . . .

M 25.1.3 Trennung von Privatem und Dienstlichem . . . . . . . . . . .

M 25.1.4 Verpflichtung zur Vertraulichkeit . . . . . . . . . . . .

M 25.1.5 Verbot rufschädigender Äußerungen . . . . . . . . . . . . .

$25.44 \quad 853$

M 25.1.6

Folgen von Rechtsverstößen . . . . . . . . . . . . .

25.48

25.54

M 25.1.7

Kontaktmöglichkeiten bei Fragen

25.56

\section{Teil 5}

\section{Verträge über internationale Datentransfers}

\section{\$26 EU-Standarddatenschutzklauseln für die Übermittlung personenbezogener Daten in Drittländer (Standardvertrag I)}

M 26.1 EU-Standarddatenschutzklauseln für die Übermittlung personenbezogener Daten in Drittländer (Standardvertrag I) - deutsch . . . M 26.2 EU-Standarddatenschutzklauseln für die Übermittlung personen-

M 26.1.1

M 26.1.2

M 26.1.3

M 26.1.4

M 26.1.5

M 26.1.6

M 26.1.7

M 26.1.8

M 26.1.9

M 26.1.10

M 26.1.11

M 26.1.12

M 26.1.13

M 26.1.14 bezogener Daten in Drittländer (Standardvertrag I) - englisch . . Abwandlung von Klausel 1 lit. a des Standardvertrags I - englisch Nummer 2 der Ergänzungsvereinbarung zum Standardvertrag I bei Aktualisierung der Anlage 1 zum Standardvertrag I - englisch Drittbegünstigtenklausel . . . . . . . . . . . . . . .

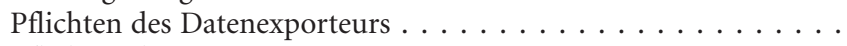


Rz. Seite

M 26.1.15 Anlage 3 zum Standardvertrag I . . . . . . . . . . . . . . . . . . . . . . . . . . . . . 26.120 916

M 26.1.16 Haftung . . . . . . . . . . . . . . . . . . . . . . . . . 26.123917

M 26.1.17 Schlichtungsverfahren und Zuständigkeit . . . . . . . . . . . . . . . 26.127918

M 26.1.18 Zusammenarbeit mit Kontrollstellen . . . . . . . . . . . . . . . . . . 26.130918

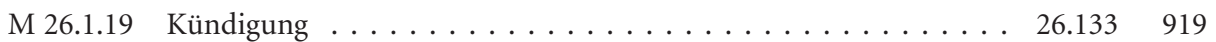

M 26.1.20 Anwendbares Recht . . . . . . . . . . . . . . . . . . . . . . . . 26.135919

M 26.1.21 Änderung des Vertrags . . . . . . . . . . . . . . . . . . . . . . . . . . 26.137920

\section{$\$ 27$ Alternative EU-Standarddatenschutzklauseln für die Übermittlung personenbezogener Daten in Drittländer (Standardvertrag II)}

M 27.1 Alternative EU-Standarddatenschutzklauseln für die Übermittlung personenbezogener Daten in Drittländer (Standardvertrag II) deutsch . . . . . . . . . . . . . . . . . Alternative EU-Standarddatenschutzklauseln für die Übermittlung personenbezogener Daten in Drittländer (Standardvertrag II) -

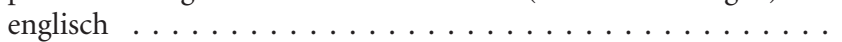

M 27.1.1

Bezeichnung der Vertragsparteien und Präambel . . . . . . . . . vertrags II ab 25.5 .2018 - deutsch . . . . . . . . . . . . . . . Abwandlung der Klausel „Begriffsbestimmungen“ des Standardvertrags II ab 25.5 .2018 - englisch . . . . . . . . . . . . . . . . . . .

M 27.1.8 Standardvertrag II: Verlagerung der Auskunftspflicht auf den Datenimporteur in der Klausel I - deutsch . . . . . . . . . . .

M 27.1.9 Standardvertrag II: Verlagerung der Auskunftspflicht auf den Datenimporteur in der Klausel I - englisch . . . . . . . . . . .

M 27.1.10 Standardvertrag II: Verlagerung der Auskunftspflicht auf den Datenimporteur durch Verweis auf Anhang B - deutsch . . . . . . .

andardvertrag II: Verlagerung der Auskunftspflicht auf de

M 27.1.12 Datenimporteur durch Verweis auf Anhang B - englisch . . . . . . .

Pflichten des Datenimporteurs . . . . . . . . . . . .

Anhang A zum Standardvertrag II . . . . . . . . . . . . . .

Haftung und Rechte Dritter . . . . . . . . . . . . .

Anwendbares Recht . . . . . . . . . . . . . .

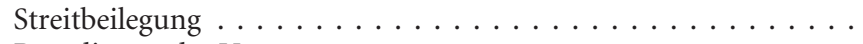

Beendigung des Vertrags . . . . . . . . . . . . . . .

M 27.1.18

Änderung der Klauseln . . . . . . . . . . . . . . . . . . . . . . . . . . . .

M 27.1.19

Beschreibung der Übermittlung $\ldots \ldots \ldots \ldots \ldots \ldots \ldots \ldots$

M 27.1.20

Anhang B zum Standardvertrag II . . . . . . . . . . . . .

M 27.1.21

Aktualisierung von Anhang B zum Standardvertrag II - deutsch . . 
$\$ 28$ EU-Standardvertragsklauseln für die Übermittlung personenbezogener Daten an Auftragsverarbeiter in Drittländern

M 28.1 Standardvertragsklausel (Auftragsverarbeiter) - deutsch . . . . . . 28.27

M 28.2 Standardvertragsklausel (Auftragsverarbeiter) - englisch . . . . . .

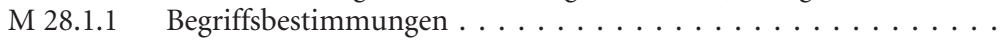

M 28.1.2 Einzelheiten der Übermittlung . . . . . . . . . . . . . . . .

$28.27 \mathrm{a} \quad 999$

M 28.1.3

Drittbegünstigtenklausel . . . . . . . . . . . . .

$28.37 \quad 1009$

M 28.1.4

Pflichten des Datenexporteurs . . . . . . . . . . . .

$28.43 \quad 1011$

$28.45 \quad 1011$

M 28.1.5

Pflichten des Datenimporteurs $\ldots \ldots \ldots \ldots \ldots \ldots$. . . . . .

28.53

1013

M 28.1.6

M 28.1.7

Haftung . . . . . . . . . . . . . . . .

M 28.1.8

Schlichtungsverfahren und Gerichtsstand . . . . . . . . . .

28.65

1016

$28.76 \quad 1018$

$28.83 \quad 1019$

M 28.1.9

Zusammenarbeit mit Kontrollstellen . . . . . . . . . . . .

28.89

1021

M 28.1.10

Anwendbares Recht . . . . . . . . . . . . . . . .

28.91

28.93

M 28.1.11

Anderung des Vertrags . . . . . . . . . . . . . . . .

28.96

M 28.1.12

Pflichten ergabe Unterauftrags $\ldots \ldots \ldots \ldots \ldots \ldots \ldots \ldots \ldots \ldots$

28.104

M 28.1.13

Anhang 1 zu den Standardvertragsklauseln . . . . . . . . . . . .

28.108

M 28.1.14

Anhang 2 zu den Standardvertragsklauseln . . . . . . . . . . . .

28.131

M 28.1.15

Beispiel für eine fakultative Entschädigungsklausel .

28.145

\section{$\$ 29$ Rahmenvertrag für EU-Standarddatenschutzklauseln}

M 29.1 Rahmenvertrag für EU-Standarddatenschutzklauseln . . . . . . .

29.61038

M 29.2

M 29.1.1

Framework Agreement for EU Standard Data Protection Clauses .

29.71046

M 29.1.2

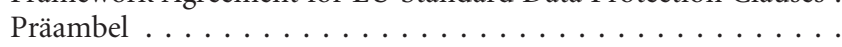

29.10

1053

M 29.1.3

Begriffe und Definitionen . . . . . . . . . . . . . . . .

29.14

1055

M 29.1.4

Anwendungsbereich und Vertragsgegenstand . . . . . . . . . . .

29.32

1059

M 29.1.5

Rangfolge und Widersprüche . . . . . . . . . . . . .

29.38

1061

M 29.1.6

Datenübermittlungen zwischen Relevanten XY-Unternehmens-

$29.42 \quad 1063$

Rechte Dritter . . . . . . $\ldots \ldots$

$29.54 \quad 1070$

M 29.1.7 Zusammenarbeit mit Aufsichtsbehörden . . . . . . . . . . . .

$29.57 \quad 1071$

M 29.1.8 Vertragsschluss, Inkrafttreten. . . . . . . . . . . . . . .

$29.60 \quad 1071$

M 29.1.9 Vertragsbeitritt, Änderungen und Beendigung . . . . . . . . . . . .

$29.66 \quad 1072$

M 29.1.10

Anwendbares Recht, Gerichtsstand

29.70

1074

\section{$\$ 30$ Vertrag zur Durchführung einer grenzüberschreitenden e-discovery}

M $30.1 \quad$ Vertrag zur Durchführung einer grenzüberschreitenden

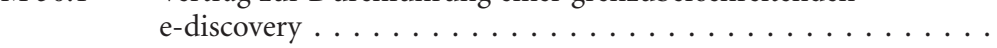

$30.16 \quad 1082$

M 30.2 Contract Regarding the Conduction of Cross-Border E-Discovery Measures . . . . . . . . . . . . . . . . . . . . 30.16a 1090

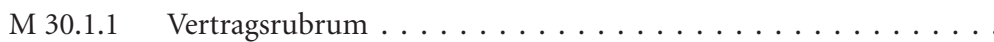

M 30.1.2

M 30.1.3

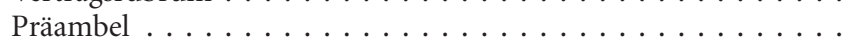

$30.28 \quad 1100$

$30.33 \quad 1101$

M 30.1.4

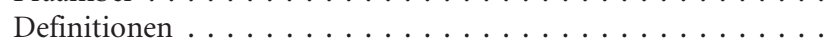

$30.36 \quad 1102$

M 30.1.5

Details der Verarbeitung. . . . . . . . . . . . . . . .

$30.39 \quad 1102$

M 30.1.6

Drittbegünstigungsklausel $\ldots \ldots \ldots \ldots \ldots \ldots$

$30.58 \quad 1108$

M 30.1.7

Pflichten des Datenexporteurs . . . . . . . . . . . . . . . .

30.631109

M 30.1.8

Pflichten des Datenimporteurs $\ldots \ldots \ldots \ldots \ldots \ldots$

$30.72 \quad 1111$

M 30.1.9

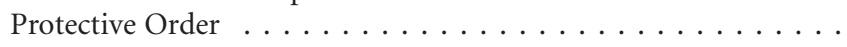

$30.92 \quad 1117$

Haftung . . . . . . . . . . . . . . . . . . . 30.971119 


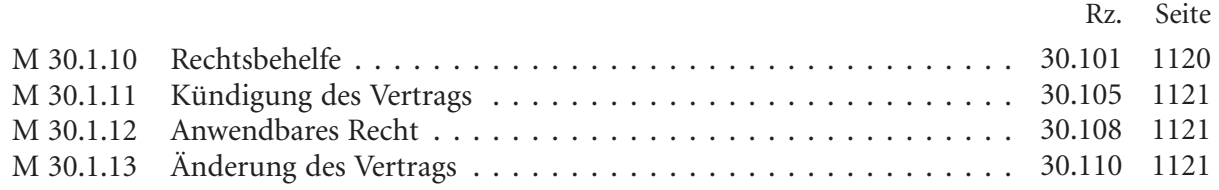

\section{$\$ 31$ Verbindliche interne Datenschutzvorschriften für Verantwortliche}

M $31.1 \quad$ Verbindliche interne Datenschutzvorschriften . . . . . . . . . . . . 31.191138

M $31.2 \quad$ Controller Binding Corporate Rules . . . . . . . . . . . . . . . . . 31.19a 1146

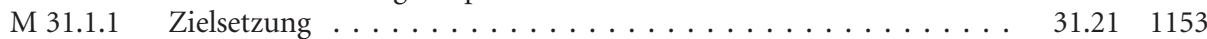

M 31.1.2 Begriffsbestimmungen . . . . . . . . . . . . . . . . . 31.301157

M 31.1.3 Anwendungs- und Geltungsbereich . . . . . . . . . . . . . . . . . . 31.361159

M 31.1.4 Grundsatz der Zweckbestimmung und Zweckbindung . . . . . . . $31.41 \quad 1161$

M 31.1.5 Rechtsgrundlagen für die Verarbeitung personenbezogener Daten . $31.47 \quad 1162$

M 31.1.6 Datenqualität und Datenverhältnismäßigkeit . . . . . . . . . . . $31.51 \quad 1163$

M 31.1.7 Transparenz und Recht auf Information . . . . . . . . . . . . . . . . . 31.561164

M 31.1.8 Rechte der betroffenen Personen . . . . . . . . . . . . . . . . . 31.611166

M 31.1.9 Sicherheit und Vertraulichkeit . . . . . . . . . . . . . . . . . 31.751171

M 31.1.10 Beschränkung der Weiterübermittlung . . . . . . . . . . . . . . . . . . 31.801172

M 31.1.11 Schulungsprogramm . . . . . . . . . . . . . . . . . . . . . . . . 31.851173

M 31.1.12 Datenschutzaudit . . . . . . . . . . . . . . . . . . . . . 31.871174

M 31.1.13 Einhaltung der BCR und Überwachung . . . . . . . . . . . . . . . 31.921176

M 31.1.14 Abweichende einzelstaatliche Vorschriften . . . . . . . . . . . . . . 31.961177

M 31.1.15 Beschwerdemöglichkeiten . . . . . . . . . . . . . . . . . . . . 31.981178

M 31.1.16 Drittbegünstigung . . . . . . . . . . . . . . . . . . . . . . . 31.1021178

M 31.1.17 Haftung . . . . . . . . . . . . . . . . . . . . . . . 31.1061180

M 31.1.18 Gegenseitige Unterstützung und Zusammenarbeit mit den

M 31.1.19 Aktualisierung der Vorschriften und Veränderungen im $\ldots \ldots \ldots . . .31 .113$. 118

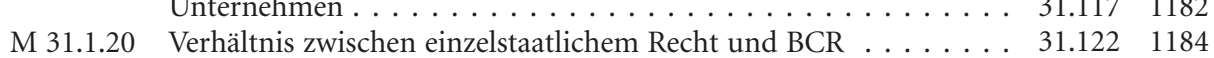

M 31.1.21 Gerichtsstand . . . . . . . . . . . . . . . . . . . . . . . . . . . . . . 31.1241184

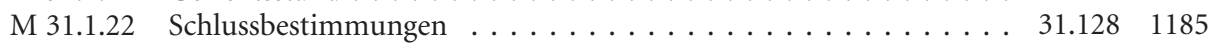

\section{$\S 32$ Verbindliche interne Datenschutzvorschriften für Auftragsverarbeiter}

M 32.1 Verbindliche interne Datenschutzvorschriften für Auftrags-

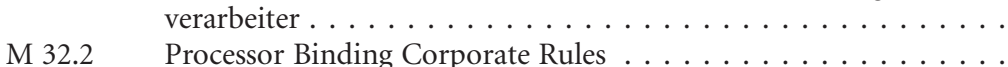

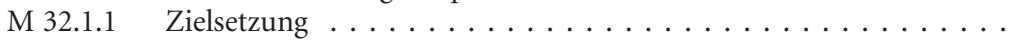

$32.13 \quad 1198$

M 32.1.2 Anwendungs- und Geltungsbereich . . . . . . . . . . . .

$32.15 \quad 1204$

M 32.1.3 Grundsatz der Zweckbestimmung und Zweckbindung . . . . . . . .

$32.21 \quad 1206$

M 32.1.4 Transparenz, Verarbeitung nach Treu und Glauben,

$32.24 \quad 1206$

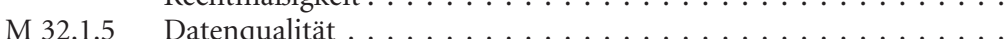

$32.29 \quad 1207$

$32.33 \quad 1208$

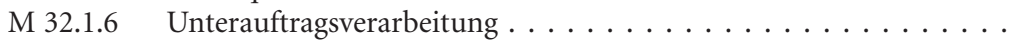

$32.37 \quad 1209$

M 32.1.7

Rechte der betroffenen Personen . . . . . . . . . . . . . . . .

$32.41 \quad 1210$

M 32.1.8

Sicherheit und Vertraulichkeit . . . . . . . . . . . . .

$32.45 \quad 1211$

M 32.1.9

M 32.1.10

Weiterübermittlung an externe Unterauftragsverarbeiter . . . . . . .

$32.51 \quad 1212$

M 32.1.11

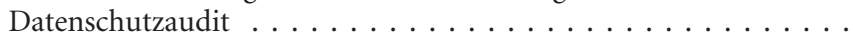

$32.57 \quad 1214$

$32.61 \quad 1215$ 


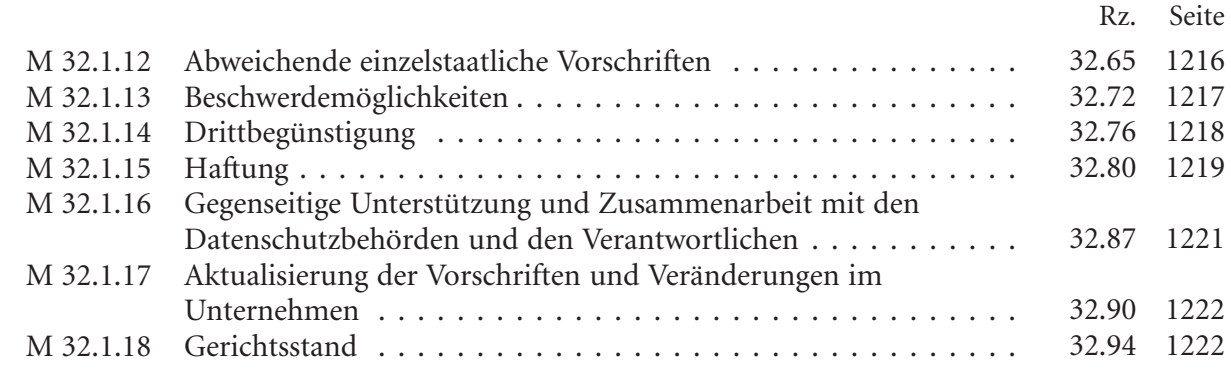

\section{Teil 6 \\ Datenschutzklauseln}

\section{\$33 Datenschutzklausel Aufgabenverlagerung (ehem. Funktionsübertragung)}

M 33.1 Datenschutzklausel Aufgabenverlagerung . . . . . . . .

M 33.1.1 Verantwortlichkeit . . . . . . . . . . . . . .

M 33.1.2 Verarbeitungsgrundsätze $\ldots \ldots \ldots \ldots \ldots \ldots \ldots \ldots \ldots$

$33.17 \quad 1233$

M 33.1.3 Technische und organisatorische Schutzmaßnahmen. . . . . . . .

$33.19 \quad 1233$

M 33.1.4 Vertraulichkeit . . . . . . . . . . . . . . .

$33.23 \quad 1234$

M 33.1.5 Prüfungs- und Kontrollhandlungen $\ldots \ldots \ldots \ldots \ldots \ldots \ldots$

$33.25 \quad 1235$

M 33.1.6

M 33.1.7

Unterauftragsverhältnisse . . . . . . . . . . . . . . . .

$33.29 \quad 1236$

M 33.1.8

Informations- und Unterstützungspflichten . . . . . . . . .

$33.33 \quad 1237$

M 33.1.9

Datenrückgabe und -löschung . . . . . . . . . . . .

$33.35 \quad 1238$

M 33.1.10

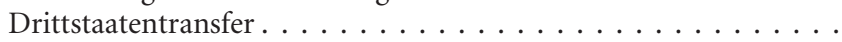

$33.37 \quad 1239$

$33.40 \quad 1239$

\section{\$34 Datenschutzklausel Kaufvertrag}

M $34.1 \quad$ Datenschutzklausel Kaufvertrag . . . . . . . . . . . .

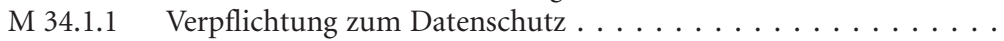

Anlass und Zeitraum . . . . . . . . . . . . . . .

Umfang der Datenerhebung . . . . . . . . . . . . . .

$34.12 \quad 1246$

M 34.1.4

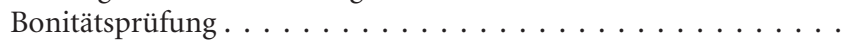

$34.14 \quad 1247$

M 34.1.5

Datenübermittlung an Dritte. . . . . . . . . . . . .

$34.20 \quad 1248$

M 34.1.6

Rechte des Betroffenen . . . . . . . . . . . .

34.23

34.25

M 34.1.7

Widerspruchsrecht . . . . . . . . . . . . . .

34.29

M 34.1.8

Verantwortliche Stelle; Aufsichtsbehörde

\section{$\$ 35$ Datenübertragungsklausel Asset Deal}

M 35.1 Datenübertragungsklausel Asset Deal $\ldots \ldots \ldots \ldots \ldots \ldots$

M 35.1.1

Vertragsgegenstand .

M 35.1.2

Übernahme von Verträgen, Verbindlichkeiten und Geschäfts-

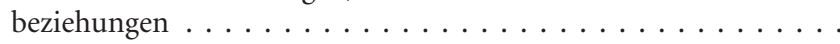

Form und Zeitpunkt der Datenübertragung . . . . . . . . . 


\section{$\$ 36$ Klauseln zur datenschutzfreundlichen Technikgestaltung}

Rz. Seite

M 36.1 Klauseln zur datenschutzfreundlichen Technikgestaltung _ . . . . 36.51277

M 36.1.1 Allgemeine Pflichten des Auftragnehmers . . . . . . . . . . . . . . 36.121281

M 36.1.2 Sicherheitsniveau/Stand der Technik . . . . . . . . . . . . . . . 36.161282

M 36.1.3 Ziele der Datenschutzmaßnahmen . . . . . . . . . . . . . . . . . . 36.251284

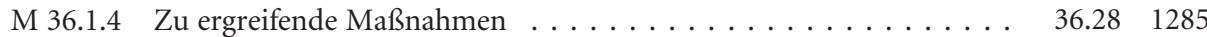

M 36.1.5 Informationspflichten und Haftungsfreistellung . . . . . . . . . . . 36.351287

M 36.1.6 Pflicht zur Vornahme datenschutzfreundlicher Voreinstellungen . . . 36.401288

M 36.1.7 Zu ergreifende Maßnahmen . . . . . . . . . . . . . . . . . . 36.471289

M 35.1.8 Zertifizierung. . . . . . . . . . . . . . . . . . . . . . 36.511290

\section{$\$ 37$ Datenschutzklausel zu EU-Angemessenheitsbeschlüssen}

M 37.1 Datenschutzklausel zu EU-Angemessenheitsbeschlüssen . . . . . . . 37.111296

M 37.1.1 Grundlage des Datentransfers aus dem EWR . . . . . . . . . . . . . . 37.131297

M 37.1.2 Unwirksamkeit des Angemessenheitsbeschlusses . . . . . . . . . . . . 37.181298

\section{$\S 38$ Klausel zur Übernahme der Datenschutzinformation}

M $38.1 \quad$ Klausel zur Übernahme der Datenschutzinformation . . . . . . . . . 38.41304

\section{Teil 7}

\section{Datenschutzerklärungen und Einwilligungen}

\section{\$39 Datenschutzerklärung für Beschäftigte}

M 39.1 Datenschutzerklärung für Beschäftigte . . . . . . . . . . . .

$39.4 \quad 1308$

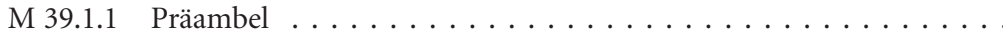

39.51313

M 39.1.2 Verantwortliche und Datenschutzbeauftragter . . . . . . . . . . . 39.81314

M 39.1.3 Quellen und Arten personenbezogener Daten . . . . . . . . . . . . 39.161316

M 39.1.4 Verarbeitungszwecke und Rechtsgrundlagen . . . . . . . . . . . . . 39.201318

M 39.1.5 Empfänger personenbezogener Daten . . . . . . . . . . . . . . 39.271321

M 39.1.6 Verarbeitungsdauer . . . . . . . . . . . . . . . . . . . . . . 39.321322

M 39.1.7 Notwendigkeit der Datenverarbeitung . . . . . . . . . . . . . . . . . . 39.371323

M 39.1.8 Betroffenenrechte . . . . . . . . . . . . . . . . . . . . . . . . . . . 39.411324

M 39.1.9 Stand und Änderung dieser Datenschutzerklärung . . . . . . . . . 39.471326

\section{$\$ 40$ Datenschutzerklärung für Geschäftskunden und Lieferanten}

M $40.1 \quad$ Datenschutzerklärung für Geschäftskunden und Lieferanten . . . . . 40.71331

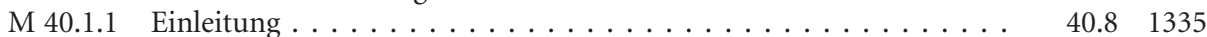

M 40.1.2 Verantwortlicher und Datenschutzbeauftragter . . . . . . . . . . . $40.12 \quad 1335$

M 40.1.3 Quellen und Arten personenbezogener Daten . . . . . . . . . . . . 40.161336

M 40.1.4 Verarbeitungszwecke und Rechtsgrundlagen . . . . . . . . . . . . 40.201338

M 40.1.5 Empfänger personenbezogener Daten . . . . . . . . . . . . . . . 40.251339

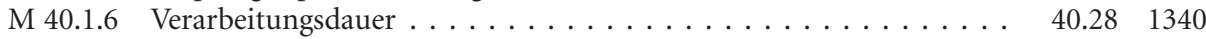

M 40.1.7 Betroffenenrechte . . . . . . . . . . . . . . . . . . 40.311341

M 40.1.8 Stand und Änderung dieser Datenschutzerklärung . . . . . . . . . 40.361342 


\section{\$41 Internet-Datenschutzerklärung}

Rz. Seite

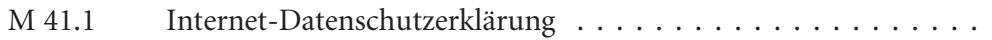

$41.20 \quad 1350$

M 41.1.1

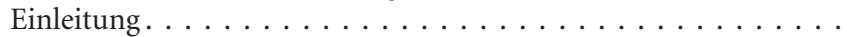

$41.21 \quad 1354$

M 41.1.2

M 41.1.3

Erheben personenbezogener Daten . . . . . . . . . . .

$41.23 \quad 1354$

M 41.1.4

Verwendungszwecke und Rechtsgrundlage . . . . . . . . . . . .

41.291357

M 41.1.5

Informationen über Ihren Computer, Cookies und Targeting . . .

41.351360

M 41.1.6

Datensicherheit . . . . . . . . . . . . . .

$41.44 \quad 1364$

M 41.1.7

Keine Weitergabe personenbezogener Daten . . . . . . . . . . .

41.521366

M 41.1.8

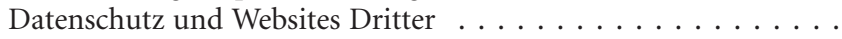

$41.54 \quad 1367$

M 41.1.9

Änderungen dieser Datenschutzbestimmungen . . . . . . . . . . . . . . . . . . . . . . . . . . . . . . . .

41.56

1367

Ihre Rechte und Kontakt . . . . . . . . . . . . . . . .

41.581368

\section{\$42 Datenschutzerklärung für Apps}

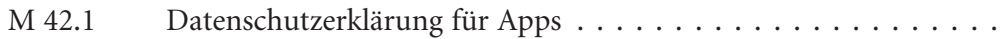

$42.6 \quad 1373$

M 42.1.1

Präambel

$42.7 \quad 1378$

M 42.1.2

M 42.1 .3

Kategorien der verarbeiteten Daten . . . . . . . . . . . . . . . . . . . . . .

$42.11 \quad 1379$

M 42.1.4

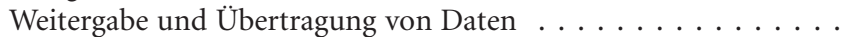

$42.20 \quad 1382$

M 42.1.5

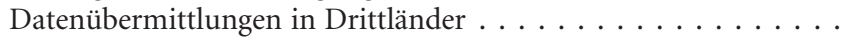

$42.26 \quad 1384$

M 42.1.6

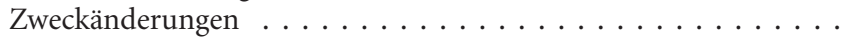

$42.31 \quad 1385$

M 42.1.7

Zeitraum der Datenspeicherung . . . . . . . . . . . . . . . . . . . . .

42.351386

M 42.1.8

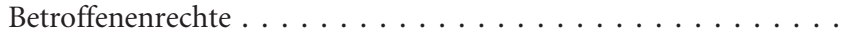

$42.38 \quad 1387$

M 42.1.9

M 42.1.10

Widerspruchsrecht $\ldots \ldots \ldots \ldots \ldots$

42.41

1388

M 42.1.11

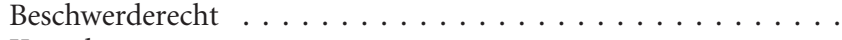

42.46

42.49

1389

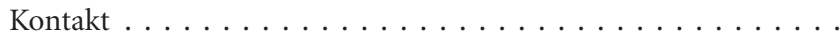

42.55

1390

Änderungen der Datenschutzerklärung . . . . . . . . . . . 1391

\section{$\$ 43$ Die SCHUFA-Hinweislösung nach DSGVO}

M 43.1 SCHUFA-Hinweis $\ldots \ldots \ldots \ldots \ldots \ldots \ldots \ldots$

$43.7 \quad 1394$

M 43.2

SCHUFA-Informationsblatt

$43.8 \quad 1395$

M 43.1.1

Umfang der Datenübermittlung . . . . . . . . . . . . . . . .

$43.16 \quad 1399$

M 43.1.2 Rechtsgrundlagen der Datenverarbeitung . . . . . . . . . .

$43.22 \quad 1400$

M 43.1.3

Befreiung vom Bankgeheimnis. . . . . . . . . . . . . .

$43.40 \quad 1403$

M 43.1.4

Profilbildung und Hinweis auf weitere Informationsquellen . . . .

43.431404

\section{$\$ 44$ Datenschutzbestimmungen und Einwilligungserklärungen für soziale Netzwerke}

M 44.1 Datenschutzbestimmungen und Einwilligungserklärungen für soziale Netzwerke . . . . . . . . . . . . . . . . . . . . . . . . 44.61412

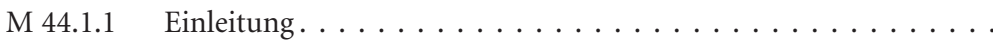

M 44.1.2 Mindestalter für die Anmeldung bei Network XYZ . . . . . . . .

M 44.1.3 Datenerhebung, Art, Zweck und Rechtsgrundlage . . . . . . . . .

$44.33 \quad 1429$

M 44.1.4

Übermittlung oder Weitergabe der Daten an Dritte . . . . . . . . .

$44.52 \quad 1435$

M 44.1.5

Schutz von Nutzerdaten . . . . . . . . . . . . . . . . . . . . .

$44.60 \quad 1439$

M 44.1.6 Änderung der Datenschutzbestimmungen . . . . . . . . . .

$44.64 \quad 1440$

M 44.1.7 Rechte der betroffenen Person . . . . . . . . . . . . . . .

$44.66 \quad 1440$

M 44.1.8 Verantwortliche Stelle, Datenschutzbeauftragter und Kontakt . . .

$44.71 \quad 1442$

M 44.1.9 Abrechnungsdaten (soziale Netzwerke) . . . . . . . . . . . . .

$44.77 \quad 1444$

M 44.1.10

Applikationen (soziale Netzwerke)

$44.80 \quad 1445$ 


\section{$\$ 45$ Werbe-Einwilligungserklärung für E-Mail- und Telefon-Werbung}

Rz. Seite

M 45.1 Werbe-Einwilligungserklärung bei Abschluss eines Vertrages:

Offline-Variante für E-Mail- und Telefon-Werbung . . . . . . . . . .

45.21448

M 45.2 Werbe-Einwilligungserklärung bei Abschluss eines Vertrages: Online-Variante für E-Mail- und Telefon-Werbung . . . . . . . . . . 45.141454

M 45.3 Werbe-Einwilligungserklärung im Rahmen eines Gewinnspiels: Offline-Variante für E-Mail- und Telefon-Werbung für mehrere Werber.......................... $45.21 \quad 1457$

M 45.4 Werbe-Einwilligungserklärung im Rahmen eines Gewinnspiels: Online-Variante für E-Mail-Werbung für mehrere Werber $\ldots \ldots \ldots \quad 45.26 \quad 1459$

\section{$\$ 46$ Einwilligung in den Einsatz nicht-essentieller Cookies}

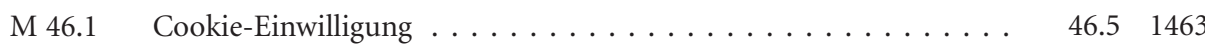

M 46.1.1 Cookie-Einwilligung - erste Ebene . . . . . . . . . . . . . . . . 46.121465

M 46.1.2 Cookie-Einwilligung - zweite Ebene . . . . . . . . . . . . . . . 46.301469

\section{$\$ 47$ Schweigepflichtentbindungserklärung}

M $47.1 \quad$ Schweigepflichtentbindungserklärung . . . . . . . . . . . . . 47.141477

M 47.2 Datenschutzrechtliche Einwilligung und Schweigepflicht-

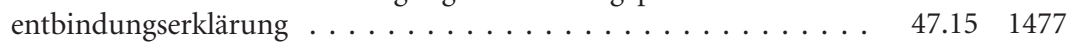

Stichwortverzeichnis . . . . . . . . . . . . . . . . . . . 1485 
